\title{
Autonomous, connected ultrasonic microsystem dedicated to monitoring the pathophysiology of the phonic and respiratory apparatus
}

\author{
MARWA SAWAN \\ Institute of Electronics, Microelectronics and Nanotechnology, Opto-Acousto-Electronic Department \\ University Polytechnic Hauts-De-France \\ Campus Mont Houy - 59313 Valenciennes Cedex 9 \\ FRANCE
}

\author{
HILAL REDA \\ Faculty of Engineering \\ Lebanese University \\ Hadath Campus \\ LEBANON \\ NADINE SAAD \\ Faculty of Science \\ Lebanese University \\ Fanar Campus \\ LEBANON \\ SUN BIN \\ College of Physics \\ Qingdao University \\ Qingdao 266071 \\ P.R CHINA
}

\section{GEORGES NASSAR}

Institute of Electronics, Microelectronics and Nanotechnology, Opto-Acousto-Electronic Department University Polytechnic Hauts-De-France

Campus Mont Houy - 59313 Valenciennes Cedex 9

\section{FRANCE}

Abstract: This work tackles a combination of two technological fields: "integrated ultrasonic biosensors" and "connected modules" coupled with "Artificial Intelligence" algorithms to provide healthcare professionals with additional indices offering multidimensional information and a "Decision Support" tool. This device comprises a connected telemedical platform (PC or Smartphone) dedicated to the objective and remote assessment of pathophysiological states resulting from dysphonia of laryngeal origin or respiratory failure of inflammatory origin.

Keywords: nanowire, ultrasonic, electroactive, tissue interfaces, vibration, telemedicine, connected platform, laryngeal dysphonia, pathophysiology

Received: April 12, 2019. Revised: December 28, 2019. Accepted: January 14, 2020. Published: February 6, 2020.

\section{Introduction}

Today, smart devices govern numerous aspects of our lives and some can even think for us. In all technological sectors, researchers are focusing on the design or optimization of operations to generate a new model of interaction between the various actors in society involved in the fields of health, comfort, and safety with a very low risk of infection [1-3]. Far from replacing the role of professionals, embedded electronic systems strengthen the links between scientists, patients, and caregivers. The 
practice of "continuous monitoring" reassures and actively involves individuals in their health. The study of interactions between sensors and living things goes far beyond biological and health impact studies. Continuous monitoring and data collection are often complicated and only a few restrictive intrusive devices (e.g. wire probes) allow continuous monitoring of a person's pathophysiology. Practices are often conditioned by health constraints, the morphology and/or age of the patient, or both, as in the case of the situation linked to the scourge of the Covid-19 epidemic. It is in this context that this paper focuses on the development of a suitable, miniaturized, autonomous, and connected prevention and diagnosis system. This system will meet the requirements of both current health constraints and increasing demand from professionals who are looking for prevention tools with a real-time, remote response.

The voice is the vector of our identity and witness of our emotions. In the current context, assessing the health of the phonatory (respiratory) apparatus and the possible causes of its degradation through different vocal indices is more than ever vital to detect fine variations in the frequency imprints, which could be an indicator of a potential disease or viral infection.

This proposal aims to implement a connected telemedical platform (Smartphone) dedicated to the objective and remote assessment of pathophysiological states resulting from dysphonia of laryngeal origin or respiratory failure of inflammatory origin. This platform primarily considers biocompatible vibration sensors for passive listening to the laryngeal apparatus. The combination of two technological fields: "integrated micro-sensors" and "connected modules" coupled with "Artificial Intelligence" algorithms will provide health professionals with additional indices offering multidimensional information and a "Decision Support" tool for assessing the pathophysiological condition representative of the symptoms identified. Our goal is:

$\checkmark$ To make the individual reactive through their contribution by providing them with the means to be more autonomous with new generations of connected wireless systems distributed in the desired environment.

$\checkmark$ To help the development of Decision Support tools by "listening to the body" with the aim of implementing an adjustment protocol according to the risks and hazards (nature and positioning of sensors, connection failure, etc.) $\checkmark$ To adapt smart mobility and therefore ensure maximum security for the individual and the information communicated

$\checkmark$ To overcome difficulties regarding coordination between professionals by developing systems of analysis and technological advances to facilitate understanding of the data and consequently adopt a common vision (common language).

\section{Physical concept}

\subsection{Tissue interfaces}

This part of the work has contributed to the evolution and development of techniques relating to the study, design, and implementation of miniaturized, autonomous acoustic/ultrasonic systems to ensure maximum contact with the skin [4-7]. Optimizing the properties of the Sensor/Tissue interface will allow better transmission of the physiological information in laryngitis dysphonia.

To quantify the response of the skin, a micromechanical model based on a finite element homogenization method was used under both static and harmonic applied loads.

The creep function associated with a pure normal stress was computed by finite element simulation of a two-dimensional Kelvin-Voigt (K-V) viscoelastic model. The general constitutive law for $2 \mathrm{D} \mathrm{K}-\mathrm{V}$ is given by:

$$
\begin{aligned}
{\left[\begin{array}{l}
\sigma_{\mathrm{xx}} \\
\sigma_{\mathrm{yy}} \\
\sigma_{\mathrm{xy}}
\end{array}\right]=\left(\begin{array}{ccc}
\lambda+2 \mu & \mu & 0 \\
\mu & \lambda+2 \mu & 0 \\
0 & 0 & 2 \mu
\end{array}\right)\left(\begin{array}{c}
\varepsilon_{\mathrm{xx}} \\
\varepsilon_{\mathrm{yy}} \\
\varepsilon_{\mathrm{xy}}
\end{array}\right) } \\
+\left(\begin{array}{ccc}
\eta_{\mathrm{v}}+2 \eta_{\mathrm{s}} & \eta_{\mathrm{s}} & 0 \\
\eta_{\mathrm{s}} & \eta_{\mathrm{v}}+2 \eta_{\mathrm{s}} & 0 \\
0 & 0 & 2 \eta_{\mathrm{s}}
\end{array}\right)\left(\begin{array}{c}
\dot{\varepsilon}_{\mathrm{xx}} \\
\dot{\varepsilon}_{\mathrm{yy}} \\
\dot{\varepsilon}_{\mathrm{xy}}
\end{array}\right)
\end{aligned}
$$

Where $\lambda, \mu$ are the Lamé coefficients, and $\eta_{v}, \eta_{s}$ are the volume and shear viscosity, respectively. When applying constant vertical shear stress

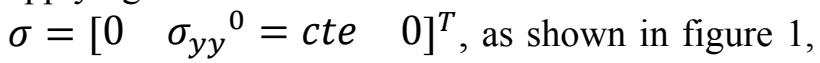
the creep functions under a constant vertical load are written as in equation (2). 


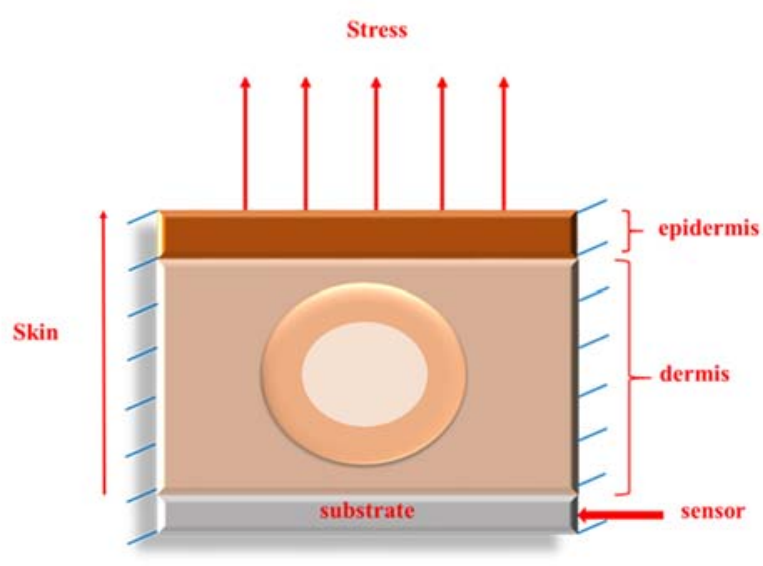

(a)

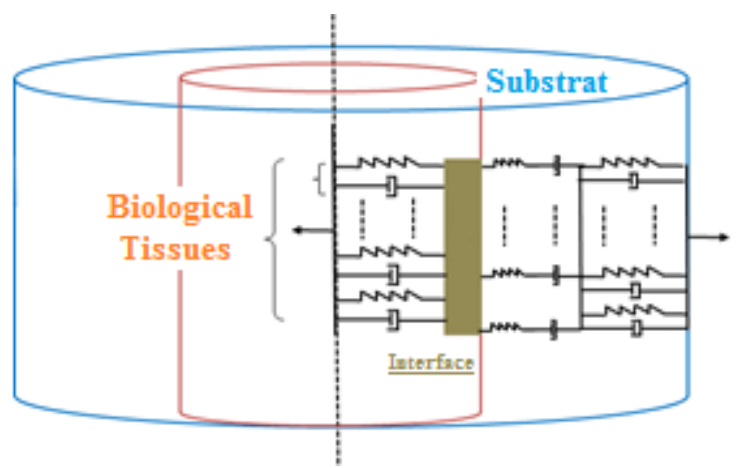

(b)

Fig.1 Vertical stress applied to the corresponding tissue unit; a) Finite element model; b) Mechanical generalized Kelvin-Voigt model.

$$
\begin{gathered}
\varepsilon_{x x}=-\frac{\sigma_{y y}}{4(\lambda+\mu)} e^{-\frac{(\lambda+\mu)}{\eta_{v}+\eta_{s}} t}+\frac{\sigma_{y y}}{4(\mu)} e^{-\frac{(\mu)}{\eta_{s}} t}-\frac{\sigma_{y y}}{4 \mu(\lambda+\mu)} \\
\varepsilon_{y y}=-\frac{\sigma_{y y}}{4(\lambda+\mu)} e^{-\frac{(\lambda+\mu)}{\eta_{v}+\eta_{s}} t}-\frac{\sigma_{y y}}{4(\mu)} e^{-\frac{(\mu)}{\eta_{s}} t}+\frac{(\lambda+2 \mu) \sigma_{y y}}{4 \mu(\lambda+\mu)} \\
\varepsilon_{x y}=0
\end{gathered}
$$

At $\mathrm{t}=\infty$, the deformation tends to a horizontal asymptote of $\frac{(\lambda+2 \mu) \sigma_{y y}}{4 \mu(\lambda+\mu)}$ at time $\mathrm{t}=5 \tau$. We present the calculation of the first Lamé coefficient $\lambda$ from the value of the maximum deformation according to equation 2 . Then, from the relation between $\lambda$ and $\mu$ given by $\frac{2 \mu \nu}{(1-2 v)}$, we can calculate the effective Poisson's ratio. The velocity of deformation is a time-dependent function. During the execution stage, the user must define an initial constraint, a time step increment, as well as the characteristics of the material used (Young's modulus, Poisson's ratio, viscosity). Boundary conditions reflect the definition of the problem. In the calculation of nodal solutions, the software provides the unknown displacement values taking into account the boundary conditions. The deformation can be calculated from the displacement history and the time step increment. From the results of the Free FEM, the deformation was plotted as a function of time to characterize the creep response within a relatively short time.

In dynamic mode, we take a general $2 \mathrm{D} \mathrm{KV}$ viscoelastic medium under normal harmonic stress $=\sigma_{0} e^{\omega t}$, where $\omega$ is the frequency. The general constitutive law for the $2 \mathrm{D} \mathrm{KV}$ is given by coupling the rigidity and viscosity matrices:

$$
\sigma(y, t)=\boldsymbol{a}(y) \varepsilon(y, t)+\boldsymbol{b}(y) \frac{\partial}{\partial t} \varepsilon(y, t)
$$

Where $\boldsymbol{a}(y)$ is the rigidity matrix and $\boldsymbol{b}(y)$ is the viscosity matrix, which represent the harmonic elastic behavior of the viscoelastic medium. Applying constant stress $\sigma_{0}$ and changing the frequency, we obtain the deformation as a function of the frequency.

\subsection{Strain Profile}

To validate the concept, this part of the study used the known physical properties of the sensor (composite) and the skin tissue comprising the epidermis and dermis.

In the model, the area of tissue under strain was 12 $\mathrm{mm}^{2}$ with two spheres of radius $4 \mathrm{~mm}$ and $3 \mathrm{~mm}$, respectively. The skin consists of a single incompressible homogeneous layer with linear viscoelastic, isotropic behavior. The study was carried out using a KV model. A vertical normal stress was applied to the tissue under strain, as shown in Fig. 1.

Table 1 shows the mechanical properties of the substrate, the epidermis, the dermis, and the membrane.

Table 1. Mechanical properties of the substrate

\begin{tabular}{lccc}
\hline & $\mathbf{E}$ (MPa) & $\boldsymbol{\eta}$ (MPa.s) & $\mathbf{9}$ \\
\hline Epidermis & 0.06 & 0 & 0.3 \\
Dermis & 0.05 & 5000 & 0.4 \\
Membrane & 0.005 & 2 & 0.4 \\
Composite & 94.103 & 0.4 & 0 \\
\hline
\end{tabular}




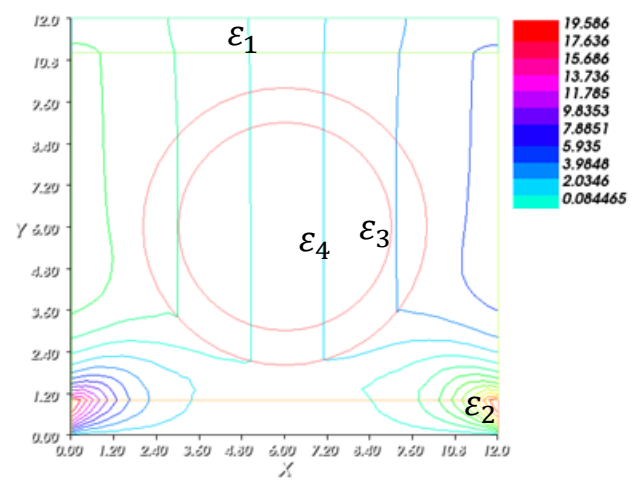

(a)

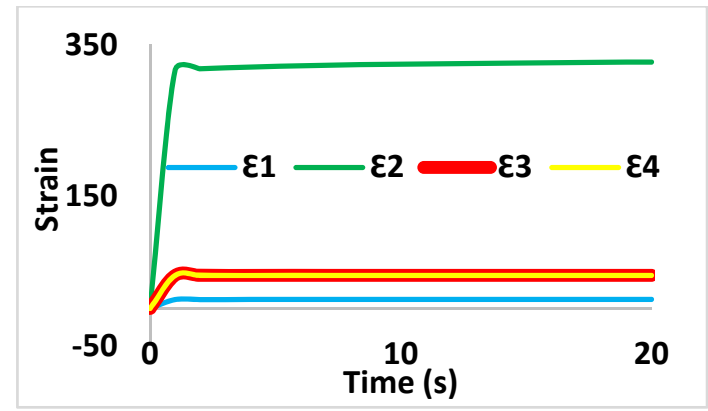

(b)

Fig.2 Variation in the (a) displacement along the y-axis as a function of time showing the deformation within the thickness of the skin and (b) the deformation as a function of time for the four regions at $t=20$ seconds and $\sigma=0.6 \mathrm{MPa}$.

The numerical approach (Free FEM) shows the internal deformations in the different regions: $\varepsilon_{1}$ at the interface between the dermis and the epidermis, $\varepsilon_{2}$ at the sensor-skin tissue interface, $\varepsilon_{3}$ at the nodule surface, and $\varepsilon_{4}$ in the hole. Regarding the boundary conditions, a vertical stress was applied to the entire tissue from region 3 to the interface with region 1 along the $x-$ and $y$-axes. Figure 2 shows the deformation in the different regions for $t=20$ seconds and a normal stress of $\sigma=0.6 \mathrm{MPa}$.

These results show that the deformation is maximal at the tissue under strain-substrate interface, the latter being considered as a sensor. Even with a maximum stress, the maximum deformation remains in the elastic region. Thus, we can tear the skin without causing any distortion inside. The variation in the deformation as a function of time for the four regions is plotted in figure 2 .

\subsection{Formalism approach}

To supplement the phenomenon of Wave/Biological tissue interactions and consequently adjust the system parameters dedicated to prevention or diagnosis, a method modeling the propagation of ultrasonic waves in multilayer biological tissues was developed from a formalism combining the physical and mechanical characteristics of the interfaces (boundary conditions) between each of the layers (Fig. 2).

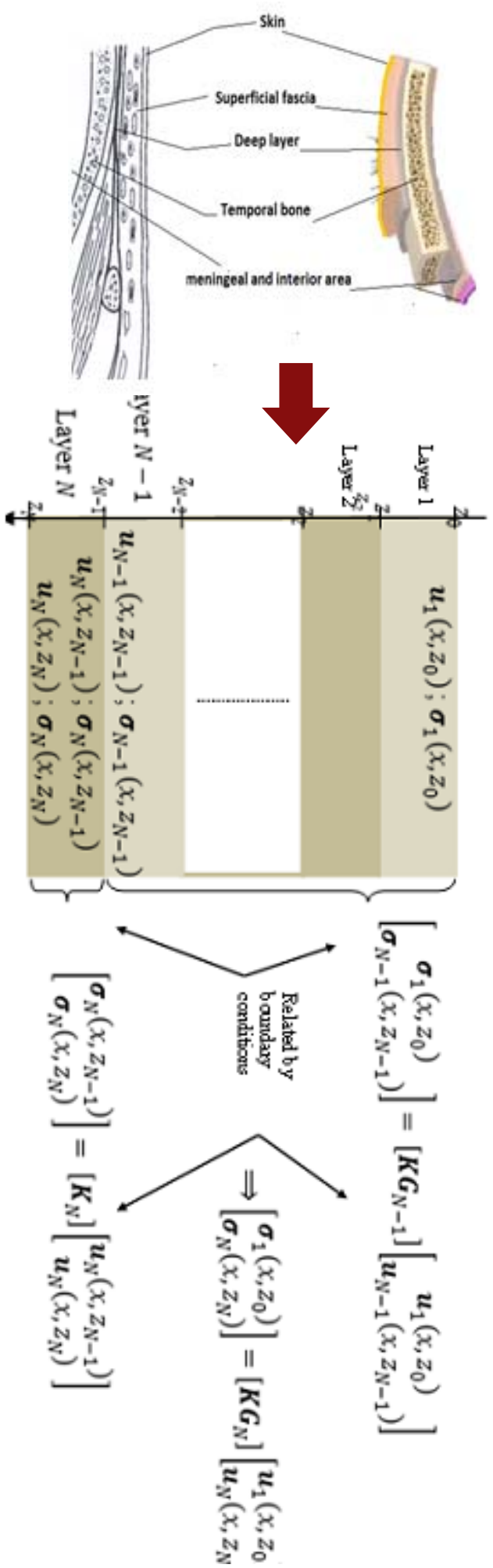

Fig. 2 Biological tissues according to a multilayer formalism [8] 


\subsection{Nanowire vibrating sensor}

Based on the work that has been carried out, this part consisted in transposing an existing collar comprising PZT elements implanted discreetly in its mass into a globally active collar by exploiting the vibratory mechanical properties of a nanowire electroactive polymer.

The curves in figure 3 show the advantage of doping electroactive polymers such as PLA and/or PVDF with $\mathrm{BaTiO}_{3}$ and GNR components on the flexibility and electrical conversion (sensitivity) of these types of sensors [9-13].
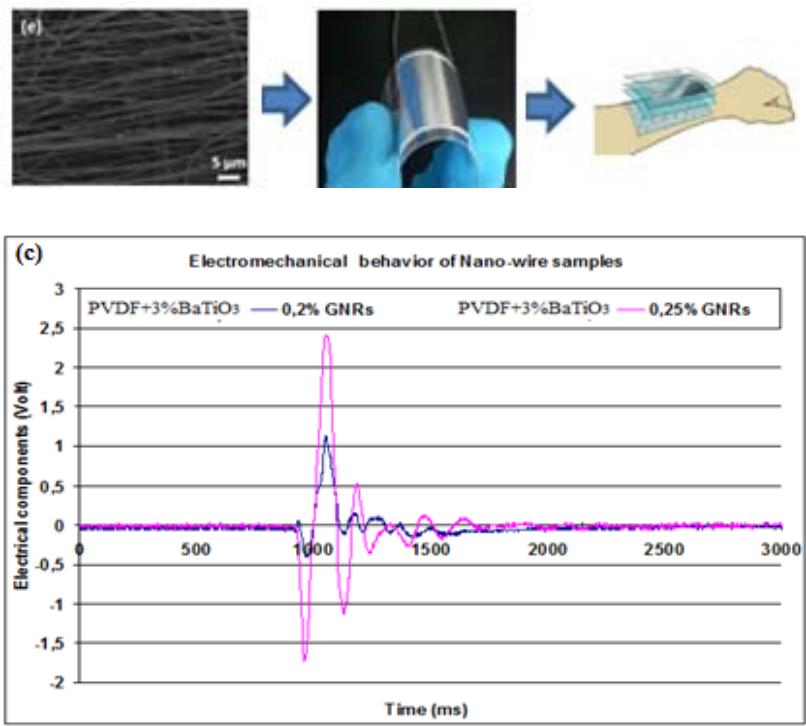

Fig. 3 Design and characterization of sensors based on flexible, optimized electroactive polymers

\subsection{Preventive passive listening microsystem}

It is a diagnostic device composed of a network of electroactive nanowire sensors covering a wide resonance frequency range $(0.1 \mathrm{KHz}-2 \mathrm{MHz})$ wired to a local CPU unit dedicated to coding, control, and analysis. This unit is coupled to a telemedical platform via a Wireless protocol for the objective evaluation and monitoring of chronic laryngeal dysphonia by means of a spectro-temporal analysis of the vibration of the vocal cords (Fig. 4). This non-invasive, non-intrusive protocol does not require the patient's physical cooperation [14-16].

The wave emanating from the mechanism of the laryngeal apparatus is transmitted through a multilayer tissue support before reception by vibratory sensors distributed adequately around the patient's neck. A matrix formalism introducing the physical properties of multilayer tissues (muscle, fat, skin) was used to determine the optimal position of the sensors. The results of the model provide the optimal dimensional parameters for pathophysiological exploration and this for a field width of 0 to $70^{\circ}$ and a frequency band of $2 \mathrm{KHz}$ (vibrational band of the vocal cords).

\subsection{Modalities of applications and analyses}

The experimental version of the intelligent collar is equipped with a bus connecting the micro-receivers to the central control unit. Signals from four different categories of one hundred patients (average age of 53 years, $20 \%$ women): healthy subjects and subjects with larynx cancer, inflammatory polyps, and chronic laryngitis, were classified after analysis by an algorithm exploiting the Time-Frequency parameters of the crossed signals (Fig. 4). The recognition rate of this approach was over $90 \%$.

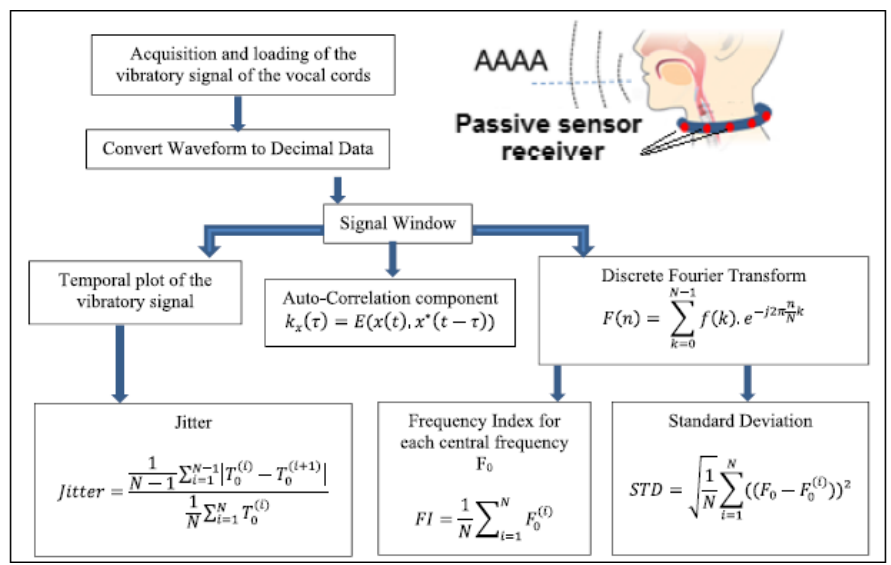

Fig. 4. Schematic illustration of the passive listening system showing the principle of setting up the multireceiver device on a collar adjusted to the patient's neck and the modalities of signal processing

The powerful information and communication techniques currently available have allowed the gradual integration of a multitude of technical platforms to form a versatile, adaptive, and scalable medical platform by transforming a computer terminal into a real physiological monitoring station in the domain of e-Health. Figure 5 shows the exchange of information over the Internet TCP / IP protocol between the patient and the doctor. 


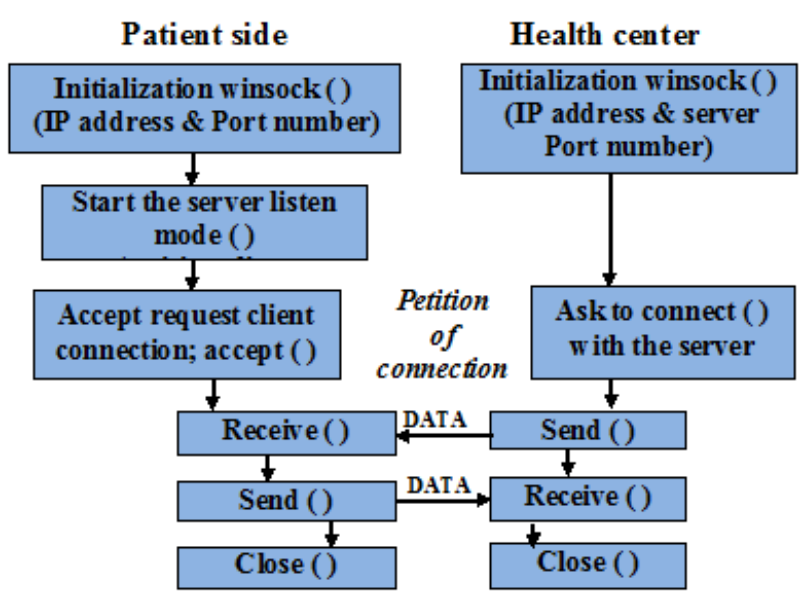

Fig. 5 "Host"/“Target" architecture

Figure 6 shows the category classification protocol in a real environment using a local graphic interface ensuring the display and communication of data through a "Host" and "Target" architecture to provide remote medical and/or health services between the patient and healthcare professionals.

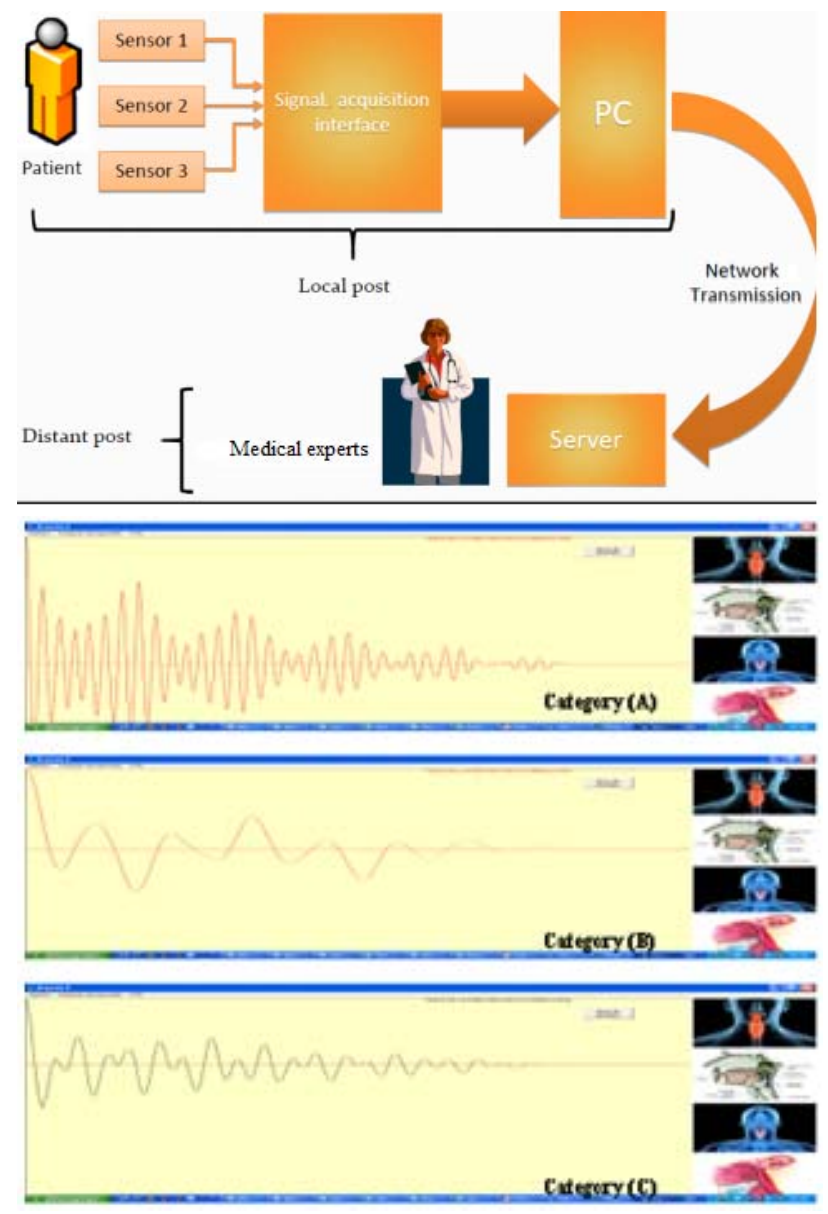

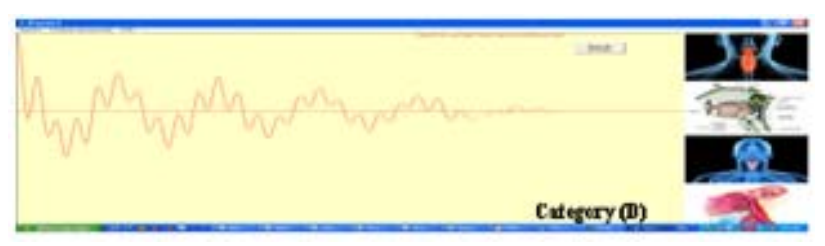

Fig. 6 Pathophysiological identification showing the four distinct pathophysiological states: (A) healthy patient, (B) cancer status, (C) inflammatory polyp, and (D) chronic laryngitis.

\section{Discussion and knowledge}

This flexible "Hard" (Fig.7a) and "Soft" (Fig. 7b) architecture combined with an artificial intelligence unit and a decision support unit could help prevent the systematic referral of patients to various specialized departments to carry out examinations and thus save precious time for both patients and physicians and, if necessary, could allow almost unlimited integration of additional examinations. These could include ECG (electrocardiograms), PTG, representative of respiratory function and respiratory muscular function, PPG, representative of circulo-respiratory function.

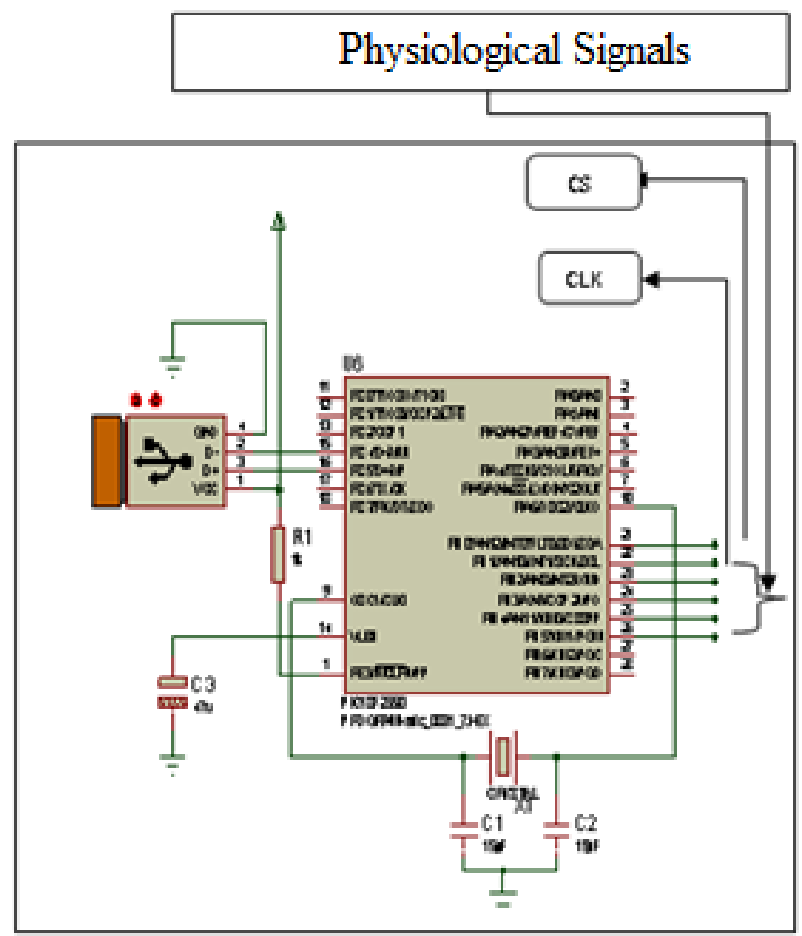

Fig.6a. "Hard" circuit ensuring the connection of the host to the peripherals via a USB terminal (local) 


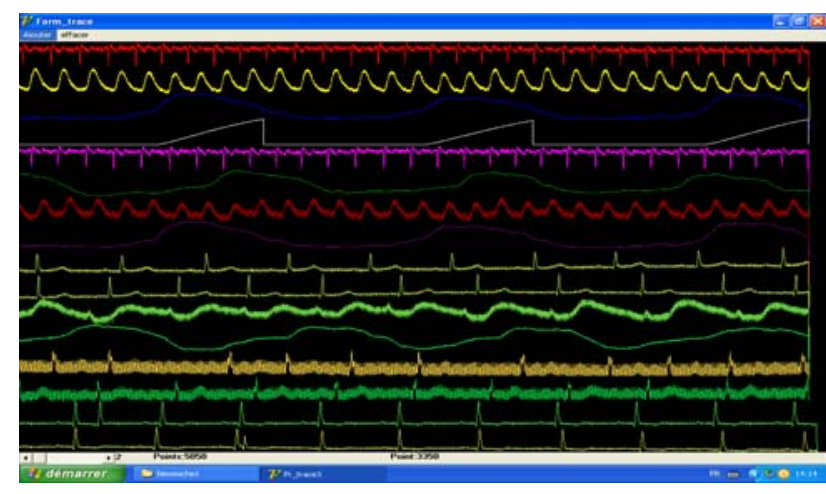

Fig. 6b "Soft" configuration showing the display of 16 distinct physiological signals obtained from a patient and transmitted remotely to a "Target" terminal of a healthcare professional (WAV transformation into HEXADECIMAL coded on 16 bits with a sampling frequency of $8 \mathrm{KHZ}$ ).

\section{Conclusion}

In this paper, we investigated the behavior of the phonatory and respiratory apparatus using an intelligent collar equipped with nanowire electroactive sensors coupled with an artificial intelligence algorithm. A micromechanical description based on a mathematical formulation was employed to evaluate the biomechanical behavior of the biological tissues. Based on this study, the most appropriate biomechanical factors were determined and quantified to help optimize the sensor/biological tissue interface conditions.

The coupling of the vibration system to a remote data transmission unit allowed the implementation of a telemedical device ensuring the remote monitoring of a patient by the physician via a secure TCP-IP communication protocol.

\section{References:}

[1] Donlan R M and Costerton J W, Biofilms: Survival Mechanisms of Clinically Relevant Microorganisms, Clinical Microbiology Reviews, Vol.15, No.2, 2002, pp. 167-193.

[2] Arciola C R, Cenni E, Caramzza R and Pizzoferrato A, Seven surgical silicones retain Staphylococcus aureus differently in vitro, Biomaterials, Vol.16, No.9, 1995, pp. 681-684.

[3] Millsap K W, Bos R, Van Der Mei H C and Busscher H J, Adhesive interactions between voice prosthetic yeast and bacteria on silicone rubber in the absence and presence of saliva, Antonie van Leeuwenhoek, Vol.79, No.3-4, 2001, pp. 337-343.

[4] Hutmacher D W, Silver Jubilee Compendium, The Biomaterials, 2000.
[5] Verran J and Boyd R D, The relationship between substratum surface roughness and microbiological and organic soiling: A review, Biofouling, Vol.17, No.1, 2001, pp. 59-71.

[6] Zinger $\mathrm{O}$ et al., Time-dependent morphology and adhesion of osteoblastic cells on titanium model surfaces featuring scale-resolved topography, Biomaterials, Vol.25, No.14, 2004, pp. 2695-2711.

[7] Lange R, Lüthen F, Beck U, Rychly J, Baumann $A$ and Nebe B, Fabrication and formation mechanism of closed-loop fibers by electrospinning with a tip collector, Biomolecular engineering, Vol.19, No.2-6, 2002, pp. 255-261.

[8] Sandrine Matta, Acoustic wave propagation in a multilayer composed of fluid, solid, and porous viscoelastic layers, INSA - HdeF, 2018

[9] Sun B, Long YZ, Chen ZJ, Liu SH, Zhang HD, Zhang JC and Han WP, Recent advances in flexible and stretchable electronic devices via electrospinning, Journal of Materials Chemistry C, Vol.2, No.7, 2014, pp. 12091219.

[10] Sun B, Jiang XJ, Zhang S, Zhang JC, Li YF, You QZ and Long YZ, Electrospun anisotropic architectures and porous structures for tissue engineering, Journal of Materials Chemistry B, Vol.3, No.27, 2015, pp. 5389-5410.

[11] Sun B, Jiang XJ, Zhang S, Zhang JC, Li YF, You QZ and Long YZ, Electrospun anisotropic architectures and porous structures for tissue engineering, Journal of Materials Chemistry B, Vol.3, No.27, 2015, pp. 5389-5410.

[12] Shi K, Sun B, Huang $X$ and Jiang $P$, Synergistic effect of graphene nanosheet and BaTiO3 nanoparticles on performance enhancement of electrospun PVDF nanofiber mat for flexible piezoelectric nanogenerators, Nano Energy, Vol.52, 2018, pp. 153-162.

[13] Guo W, Tan C, Shi K, Li J, Wang XX, Sun B, Huang $\mathrm{X}$, Long $\mathrm{YZ}$ and Jiang $\mathrm{P}$, Wireless piezoelectric devices based on electrospun PVDF/BaTiO3 NW nanocomposite fibers for human motion monitoring, Nanoscale, Vol.10, No.37, 2018, pp. 17751-17760.

[14] Nemmich A, Benabdallah M, Hamlil M, Rerbal $\mathrm{S}$ and Nassar G, Development of a platform dedicated to the telemedical practice under local architecture USB-HID and distant TCPIP, International Journal of Engineering \& Technology, Vol.15, No.3, 2015, pp. 20-27.

[15] Sandrine Matta, Daher Diab, Nadine Saad3, Dany Ishac, Georges Nassar, "Passive wideband concentric rings resonator for vocal 
cords abnormalities detection: Application on Larynx Cancer Pathologies", Review of Scientific Instruments, Vol. 91, No.1, 2020, pp. 015115-1-9

[16] Fayssal Hamza Cherif, Lotfi Hamza Cherif, Mohammed Benabdellah, Georges Nassar, "Monitoring driver health status in real time", Review of Scientific Instruments, Vol. 91, No.3, 2020, pp. 035110 1-7. 